\title{
Urbanizaciones cerradas y agua: Conflictividad en San Miguel de Santo Domingo de Heredia (2012-2018)
}

\author{
Fabricio Montagnini Salazar
}

Artículo

Afiliación: Independiente, San José, Costa Rica

E-mail: f.montagnini15@gmail.com

Recibido: 12 de marzo del 2019

Aceptado: 12 de abril del 2019

\section{Fabricio Montagnini Salaza}

Bachiller y Licenciado en Sociología por la Universidad de Costa Rica. Becario del Instituto de Investigaciones Sociales de la Universidad de Costa Rica.
1 El artículo proviene de los resultados de la tesis Gestión del agua en Costa Rica: Estudio de caso de la conflictividad en San Miguel de Santo Domingo de Heredia (2011-2018). Dicha investigación fue financiada por el Instituto de Investigaciones Sociales de la Universidad de Costa Rica por medio de su programa de becas de investigación.

\section{Resumen:}

El presente artículo muestra una serie de acciones colectivas comunales que se han opuesto a urbanizaciones cerradas en San Miguel de Santo Domingo en la provincia de Heredia. La carta principal de oposición es el impacto en los acuíferos y la utilización del agua por parte de este tipo de proyectos. La finalidad del artículo es mostrar como una serie de relaciones de poder entre actores económicos, políticos y comunales inciden en la forma de gestionar el recurso. Para esto, se entrevistó a distintos actores comunales, además de una revisión de noticias y documentos institucionales para narrar y comprender el conflicto en la zona. La información recabada muestra como las urbanizaciones cerradas, acompañadas de un aval del gobierno local, han permitido que se presenten alianzas público-privadas para la gestión del agua, incluso en una zona de protección especial ante este tipo de construcciones. Se concluye que este caso es un reflejo estructural de los cambios recientes en el uso de suelo a nivel provincial con el paso de cafetales a una renta inmobiliaria, pero que encontró resistencia ante actores comunales que han experimentado problemáticas de escasez y contaminación hídrica, así como una tensión entre visiones de mercado en contraste con un carácter público del agua.

Palabras clave: acciones colectivas; conflicto del agua; municipalidad; poder; urbanizaciones cerradas

Gated communities and water: Conflict in San Miguel de Santo Domingo de Heredia (2012-2018)

\section{Abstract:}

This article is based on collective actions against gated communities in San Miguel, Santo Domingo of Heredia. The main reason for opposition is the impact on aquifers and the use of water by this kind of urbanizations. The purpose of the article is to show how the power relations between economic, political and community actors impact the manner of managing the water. For this reason, different community actors were interviewed, as well as a review of news and institutional documents to narrate and understand the conflict in the area. The information collected shows how gated communities, accompanied by a guarantee from the local government, have allowed public-private alliances for water management, even in a special protection area for this kind of construction. In conclusion, this case is a structural impact of recent changes in land use at provincial level with the passage of coffee plantations to a real estate income, but it found the resistance of community actors who have experienced problems of scarcity and water pollution as well as a tension between market visions in contrast to a use public of water.

Keywords: collective actions; gated communities; municipality; power; water's conflict. 


\section{Introducción}

lestudio de caso que se expone en esteescrito busca reflexionar en torno ala conflictividad por la gestión del agua. Esto ante un proceso de neoliberalización de la misma en el que se intenta posicionar el líquido como un recurso con valor económico, o bien, al servicio de actividades de acumulación que necesitan del agua para desarrollarse. En el país, estos procesos suelen estar acompañados de vacíos legales con poca claridad de funciones y responsabilidades de las instituciones relacionadas con el manejo del agua (Alpizar, 2012).

Tal panorama ha incidido en la emergencia de múltiples conflictividades, donde estas son un reflejo de relaciones de poder entre actores económicos, institucionales, sociales y comunales que moldean la forma en que se gestiona el agua. En esta línea, los antecedentes de estudios de conflictos por el agua en Costa Rica son predominante en zonas costeras con proyectos hoteleros que buscan abastecerse del recurso o bien en lugares con monocultivos [piñeras principalmente] que contaminan las fuentes hídricas (Bolaños, 2016; Fenell, 2013; Navas \& Cuvi, 2015; Ramírez 2008; Silva, 2016).

De este modo, es poco lo que se ha abordado acerca de las conflictividades en ámbitos urbanos, por lo que este texto está guiado con la interrogante: ¿Cómo las relaciones entre actores económicos, institucionales y comunales en zonas sometidas a procesos de urbanización inciden en la gestión del agua?

El caso de San Miguel en el cantón Santo Domingo de Heredia presenta características asociadas con tal cuestionamiento porque se ha presentado una contraposición de intereses entre desarrolladores que buscan llevar a cabo urbanizaciones cerradas que requieren abastecimiento hídrico (residenciales, condominios, torres de apartamentos), actores comunales que se han opuesto a tal esquema y actores institucionales que han sido permisivos ante tales proyectos en una zona especial de protección y de recarga acuifera.

La oposición vecinal ha incidido en generar algunas moratorias y restricciones ante tal esquema de desarrollo, sin embargo, varias de estas construcciones han sido aprobadas incluso con acuerdos en los que el municipio de Santo Domingo les ha concesionado pozos, reflejando alianzas público-privadas que no han sido del agrado para tales sectores comunales. Esto no se ha presentado de manera aislada, ya que lo anteceden una serie de deficiencias de potabilidad, abastecimiento y permisos para actividades industriales que pueden impactar los cuerpos de agua de la zona.

Tales situaciones son parte del contexto que se expone más adelante, esto para posteriormente describir la conflictividad registrada entre el 2012 al 2018 sobre la que versa el artículo, así como una serie de reflexiones a partir de lo recabado. A continuación, se exponen las bases teóricas con las que se analizó el caso y la forma metodológica de llevar a cabo este proceso investigativo.

$2 \quad$ Existen posicionamientos como el de Lukes (2007) con su enfoque radical y multidimensional del poder en el que los agentes buscan influir en los intereses de otros; Elias (2008) sobre las cuotas de poder de cada individuo en toda relación social; Castells (2009) al definir el poder como la capacidad relacional en la que un actor social influye asimétricamente en las decisiones de otros para hacer valer sus intereses.

\section{Referentes conceptuales: ¿Cómo entender las relaciones sociales en torno la gestión del agua?}

Se considera que el intento de gestionar el agua se enfrenta a una multiplicidad de intereses que surgen de relaciones de poder, las cuales son clave para entender el posicionamiento de los actores involucrados. Una definición básica del mismo se obtiene con Weber (2002) quien define poder como la probabilidad de imponer la voluntad propia dentro de una relación social, incluso si existe resistencia, mientras que por dominación entiende la probabilidad de encontrar obediencia ante un mandato.

Aun así, las reflexiones sobre el poder son múltiples ${ }^{2}$ por lo que otra definición importante se encuentra en Foucault quien abandona una corriente que considera el poder como algo que unos poseen y otros carecen, y lo posiciona en términos relacionales:

Lo que es decir, desde luego, que no existe algo llamado el Poder, o el poder, que existiría universalmente, en forma masiva o difusa, concentrado o distribuido. Sólo existe el poder que ejercen "unos" sobre "otros". [Éste es] "(...) Un conjunto de acciones sobre otras acciones. (Foucault, 1988, p.14-15) 
Al reflexionar tales relaciones de poder en torno a cuestiones que involucran la naturaleza, entra en juego la ecología política al considerar que tales relaciones median la interacción sociedad-ambiente, por lo que las problemáticas en ámbitos locales como el caso en estudio, están condicionadas por factores económicos y sociopolíticos de una escala más amplia (Clare \& Meléndez, 2012).

A partir de esto, se puede considerar que la situación de San Miguel refleja un conflicto socioambiental, en el que el manejo de los recursos naturales genera oposiciones entre actores (Walter, 2009), donde tales diferencias poseen su origen en distintas valoraciones, percepciones y significados de las acciones sobre el ambiente y que son expresadas por medio de acciones colectivas en el ámbito público (Gudynas, 2014).

Sin embargo, no se debe perder de vista que tal conflictividad es el reflejo de un tejido social más amplio, en el que las relaciones sociales de producción y mercantiles, la explotación ya sea de fuerza de trabajo humana y de los recursos naturales, y un contexto de desigualdad social en diferentes esferas (de ingresos, territorial, género, étnico-racial) inciden en la forma que adquieren los conflictos sociales relacionados con el ambiente.

Esto obliga a repensar las conceptualizaciones sobre la forma de gestionar el agua, ya que generalmente se conceptualizan tres tipos. Una de estas es la gestión jerárquicatradicional caracterizada por un manejo público-estatal con poca participación de otros actores (Instituto Costarricense de Acueductos y Alcantarillados (ICAA) y municipalidades). Otro modelo es la gestión privada-mercantil regida por una lógica de acumulación a cargo de entes privados (Alianzas público-privadas). El tercero de estos modelos es la gestión social-comunal caracterizada por una administración sin fines de lucro con relaciones de cooperación y solidaridad. Como las Asociaciones Administradoras de Sistemas de Acueductos y Alcantarillados Sanitarios (ASADAS). (Alpízar 2012; Bolaños, 2016).

Por último, se debe considerar que tales conceptualizaciones de gestionar el agua forman parte de una tipología ideal ${ }^{3}$, por lo que se pueden presentar entremezcladas en la realidad. Esto se manifiesta por medio de acuerdos entre entes públicos y privados para administrar el recurso, lo que refleja la preocupación de autores como Nora Rabotnikof (2005), ya que, históricamente lo público ha recaído sobre el Estado y sus instituciones, pero el deterioro de estas organizaciones, ha incidido para que otro tipo de agrupaciones de carácter privado asuman estas funciones como se repasará más adelante.

\section{Aspectos metodológicos}

3 Los tipos ideales se caracterizan por ser la construcción de un cuadro a partir de rasgos comunes que se repiten en la realidad pero que no se presentan tal cual en la misma. (Weber, 2002)

$4 \quad$ Disponible en http://banderazulsmiguel. blogspot.com/.

$5 \quad$ Disponible en https://www.facebook.com/ Tierra-Miguele\%C3\%B1a-143240702355754/
La metodología del presente artículo fue de índole cualitativa, con la cual se busca acercarse a los detalles del fenómeno social seleccionado para investigar (Ragin 2007). De esta manera, el presente documento se construyó a partir de una revisión de documentos que incluyen informes y resoluciones de entes estatales, actas, resoluciones e informes de la Municipalidad de Santo Domingo, investigaciones académicas, así como revisión de prensa que ha dado seguimiento al caso (La Nación, CrHoy.com, Semanario Universidad y Prensa Libre).

Además, para acceder a la perspectiva comunal del conflicto se accedió al blog del Comité Bandera Azul Ecológica de San Miguel ${ }^{4}$, la página de Facebook Tierra Migueleña ${ }^{5}$ en la que el Comité Conservación Cuenca del Río Virilla (CCCRV) emite sus comunicados, así como dos entrevistas semi-estructuradas a líderes y lideresas comunales del distrito involucrados en el proceso y una a un exregidor municipal que también es vecino de la localidad.

A esto se deben sumar dos observaciones participantes, la primera en una rendición de cuentas del alcalde de la Municipalidad de Santo Domingo en la Escuela Presbítero Ricardo Salas en San Miguel el día 26 de abril del presente año, y la segunda de estas el 16 de mayo en una exposición-conversatorio organizado por el CCCRV en el auditorio del Colegio Lincoln en Barrio Socorro, a la cual asistieron vecinos, el alcalde, regidores y síndicos. 
Tabla 1. Cantidad total y porcentaje de habitantes en Heredia dividido por año y tipo de población.. Fuente: Elaboración propia a partir de Censos Poblacionales del INEC (1984; 2000; 2011)
$6 \quad$ Estos cambios de uso del suelo se aprecian en todos los cantones de Heredia que forman parte del sector norte del Gran Área Metropolitana (GAM): Heredia, Barva, Santo Domingo, Santa Bárbara, San Rafael, San Isidro, Belén y San Pablo. Sin embargo, Santo Domingo en conjunto con Heredia centro y Belén, son los cantones con modificaciones mayormente acentuadas.
Es así como las noticias consultadas, las entrevistas y los recursos electrónicos de los actores comunales permitieron realizar una reconstrucción cronológica del conflicto. Por su parte, los documentos institucionales y las observaciones participantes en las que asistieron funcionarios del municipio permitieron acceder al posicionamiento de estos ante la situación y la gestión que han llevado a cabo.

\section{Contextualización: Los cimientos de una disputa por el agua}

El caso de San Miguel, distrito del cantón Santo Domingo en Heredia, presenta características de una zona en proceso de urbanización la cual es una dinámica que se ha generado a nivel provincial desde la segunda mitad del siglo XX (Monge, 1987), lo que ha traído consigo cambios en la zona, así como mayor demanda de recursos, entre ellos el agua.

Los cambios en el uso de suelo en Heredia han estado asociados a un paso de actividades agrícolas, principalmente cafetales, a actividades de renta inmobiliaria. Al situarse en el cantón de Santo Domingo, se aprecia que el área cultivada con café decayó de 1459 hectáreas en 1984 a 929 hectáreas en el 2004. En contra parte, la extensión de área construida en hectáreas presentó un incremento de 412 a 851 de 1984 al 2010, es decir, se duplicó (Alvarado \& Pérez Sáinz, 2012). ${ }^{6}$

Esto a su vez se ha reflejado en un incremento poblacional en la provincia y cambios en el carácter de la misma. En Heredia, a partir de la década de los cincuenta se dio un paso de 50.000 habitantes a casi 200.000 en la década de los ochenta (Monge, 1987), a partir de ese momento la tendencia de incremento poblacional se ha mantenido, lo cual se aprecia en el siguiente cuadro:

\begin{tabular}{|lccccccc|}
\hline \multicolumn{1}{|c}{ Año } & 1984 & & 2000 & & \multicolumn{2}{c}{2011} \\
\hline & Cantidad & $\%$ & Cantidad & $\%$ & Cantidad & $\%$ \\
$\begin{array}{l}\text { Población } \\
\text { urbana }\end{array}$ & 98878 & 50,05 & 241790 & 68,16 & 372883 & 85,98 \\
$\begin{array}{l}\text { Población } \\
\text { rural }\end{array}$ & 98697 & 49,95 & 112942 & 31,84 & 60794 & 14,02 \\
$\begin{array}{l}\text { Población } \\
\text { total }\end{array}$ & 197575 & 100 & 354732 & 100 & 433677 & 100 \\
\hline
\end{tabular}

La Tabla 1 muestra que la población en Heredia ha aumentado en términos cuantitativos, ya que ésta se duplicó de la década de los ochenta al primer decenio de los 2000. No obstante, el tipo de población categorizada como rural y urbana pasó de compartir porcentajes en 1984, a que la de carácter urbano se convirtiera en mayoría con un $85 \%$ en el 2011. Específicamente para San Miguel, el censo del 2011 registró aproximadamente 6300 habitantes cuando en 1984 apenas pasaba los 3000 habitantes (INEC, Censos 1984 y 2011).

Ante este cambio del uso del suelo y el respectivo incremento poblacional, se debe aclarar que geográficamente San Miguel limita al sur con San Juan de Tibás y al sureste con San Vicente y la Trinidad de Moravia, distritos en los que la mancha urbana es más pronunciada como se aprecia en la Figura 1. Además, el distrito es atravesado por la carretera Braulio Carrillo (Ruta 32) que es la principal ruta que conecta la Región Central con el Caribe. Esto convierte la zona en lo que comúnmente se conoce como "ciudad dormitorio" a raíz del fácil acceso a la capital por su cercanía y por la carretera mencionada (Agüero, Pérez \& Pujol, 2014). 
Figura 1. Imagen satelital del distrito de San Miguel de Santo Domingo de Heredia. (2018). Fuente: Mapas sociales del INEC.

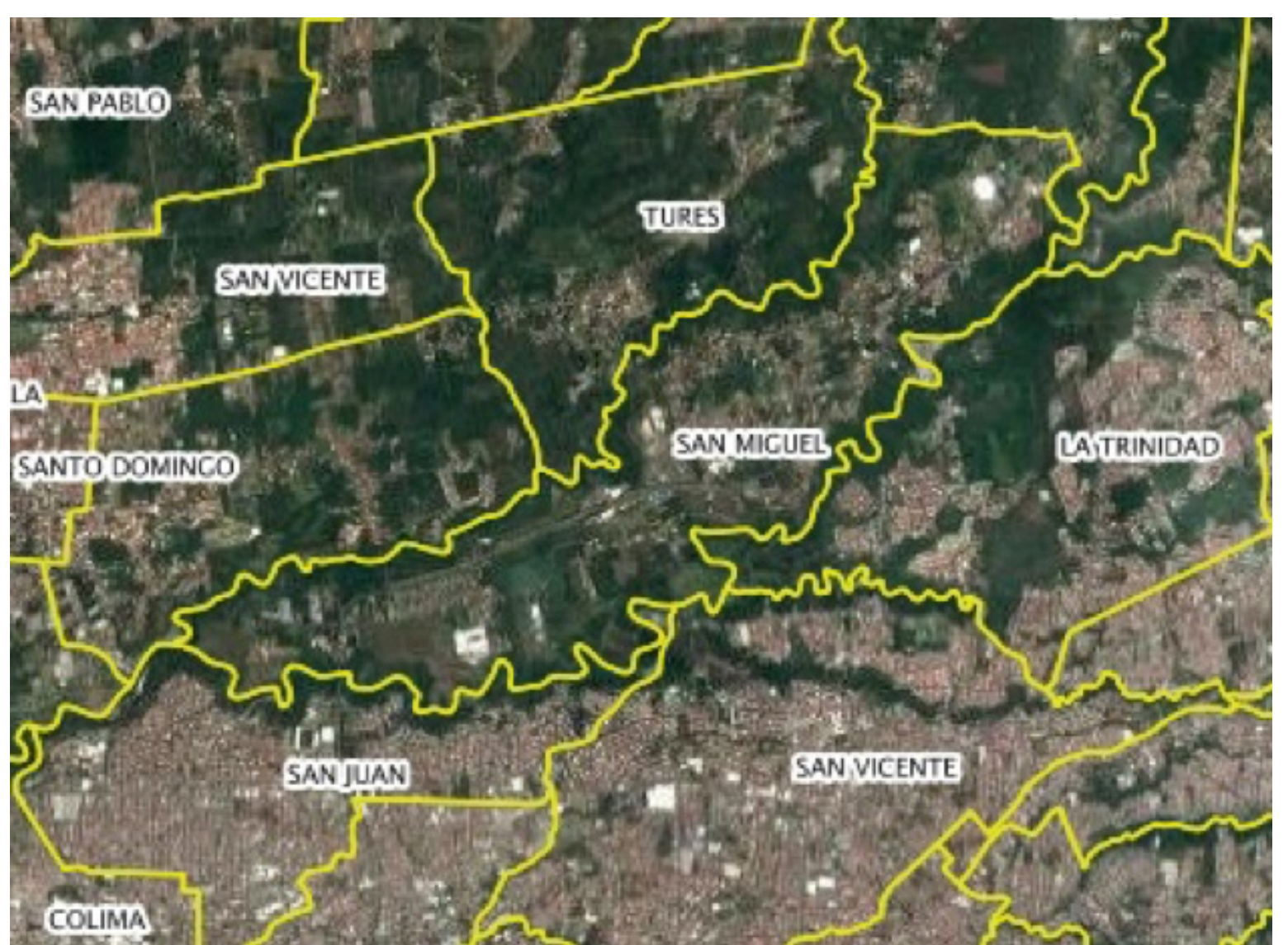

Por otra parte, al considerar el tema del agua, se debe agregar que uno de los límites naturales de San Miguel es el Río Virilla, que lo divide de los cantones Tibás y Moravia. Además, en Santo Domingo existen 98 pozos para uso de agua con un promedio de 3,5 pozos por $\mathrm{km}^{2}$ (Vargas, 2006) mientras que en el distrito transcurren los acuíferos Barva, Colima Inferior y Colima Superior (Madrigal, Fonseca, Nuñez \& Gómez, 2014).

Estos datos reflejan la disponibilidad de fuentes hídricas en la zona, sin embargo, esto no es sinónimo de consenso en torno al tipo de gestión en el distrito ni de ausencia de problemáticas por el agua. Un antecedente claro son los problemas de abastecimiento y potabilidad que han afectado a San Miguel, donde apenas en el 2015 entró en funcionamiento una planta para potabilizar el agua (Oviedo, 2015).

Antes de ese año los cortes del líquido se presentaban con mayor frecuencia e incluso existieron problemas por contaminación fecal. En el 2011 hubo un brote de diarrea que afectó a habitantes de San Miguel, Paracito, Pará y Tures. Esto ocurrió debido a que el agua se tomaba de los ríos cercanos y era clorada por medio de pastillas, método que no fue suficiente para detener tal contaminación. A causa de esto, se presentó un cierre temporal de centros educativos, más de 110 personas fueron atendidas por la clínica de Tibás, y una serie de manifestaciones de los vecinos a raíz de la problemática (Fonseca, 2011a; Fonseca, 2011b).

El Comité Bandera Ecológica de San Miguel denominó tal situación como la "Crisis del agua en los distritos del Este (de Santo Domingo)". 7 El descontento vecinal fue enfocado hacia la Municipalidad de Santo Domingo, que es la encargada de gestionar el agua en la zona, esto en un panorama donde a nivel nacional las municipalidades y las ASADAS son los entes que poseen menos capacitación técnica y recursos económicos para administrar el líquido (Angulo, 2015).

A esto se debe sumar una institucionalidad difusa en la que ocasionalmente se traslapan funciones y no hay claridad de qué le corresponde a cada ente. Además, la ley de aguas actual fue promulgada en 1942, donde al pasar los años han entrado en vigor nuevos reglamentos y leyes que reformulan aspectos de dicha ley ${ }^{8}$ (Alpízar, 2012; Alvarado, Avendaño, Blanco \& Rodríguez, 2004).

En el caso de los gobiernos locales, la legislación establece un mínimo de responsabilidades para gestionar el agua: administrar el recurso, proteger los cuerpos de agua en terrenos municipales, abastecer de agua potable a la población, asegurar zonas de protección de fuentes hidrográficas, otorgar patentes y permisos, cancelarlos en caso de contaminación, y resolver conflictos en torno al agua (Alvarado et. Al., 2004). Asimismo, cada municipalidad puede contar con su propio reglamento con la posibilidad del contrato de servicios (Alpízar, 2012). 
9 Según la Ley de Planificación Urbana $\mathrm{N}^{\circ}$ 4220 (1968) este es el "instrumento de planificación local que define en un conjunto de planos, mapas, reglamentos y cualquier otro documento, gráfico 0 suplemento, la política de desarrollo y los planes para distribución de la población, usos de la tierra, vías de circulación, servicios públicos, facilidades comunales, y construcción, conservación y rehabilitación de áreas urbanas". Dicho plan forma parte de una lógica de ordenamiento territorial que pretende dar un uso sostenible de los espacios geográficos (Astorga, 2011)

\section{Comité vecinal que se ha opuesto a} plantas de asfalto y proyectos urbanísticos en el distrito.

11 El MOPT (2010) establece que las plantas de asfalto deben desarrollarse en zonas con poca cobertura boscosa, lejos de zonas de recarga y mantos acuíferos, así como áreas pobladas, situaciones que son incumplidas.

\section{Esto aún ante resoluciones de la} Secretaría Técnica Ambiental (SETENA), el Tribunal Ambiental Administrativo (TAA), el Instituto Geográfico Nacional (IGN) y el conocimiento de las Municipalidades de Santo Domingo y Moravia (Solís, 2017).

13 Información constatada en entrevista a lideresa comunal de San Miguel el 1 de junio del 2018.
Otro elemento es que la Municipalidad de Santo Domingo no cuenta con un Plan Regulador ${ }^{9}$ y donde uno de los miembros del Comité Conservación Cuenca del Río Virilla (CCCRV) ${ }^{10}$ aduce que las peticiones del comité:

(...) se resumen en un plan regulador, (...) esa es la principal, regulación para este riego de condominios y caseríos que están saliendo por todo lado sin ningún orden y el caos vial. Muchos solo vemos las casas, pero multiplique de 600 a 800 apartamentos que no son para clase baja. (Líder Comunal del CCCRV, comunicación personal, 18 de julio, 2018)

Ante tales condiciones, se configura un contexto en el que el surgimiento de conflictos de este tipo no es sorpresa, en donde el intento de gestionar el agua se enfrenta a una multiplicidad de intereses que se resume en relaciones de poder que son clave para entender el posicionamiento de los actores involucrados. Todo esto con desarrolladores que buscan llevar a cabo sus proyectos ante una resistencia de algunos sectores comunales que se oponen, y una institucionalidad que se posiciona en medio de tales intereses.

\section{La conflictividad por el agua en concreto ¿Qué ha ocurrido en San Miguel ante el proceso de urbanización?}

Como se mencionó anteriormente, la problemática por la gestión del agua en San Miguel estaba relacionada con el abastecimiento y la potabilidad aún hasta el 2015 con la apertura de la planta potabilizadora. De esta manera, la conflictividad se apaciguó en torno a la administración técnica del agua, sin embargo, ésta se trasladó a otras esferas relacionadas con la protección de las fuentes hídricas.

Además, la localidad ha resultado atractiva por su cercanía a otras zonas de Heredia y San José, esto combinado con la disponibilidad de espacio para urbanizar, desarrollar proyectos económicos y el acervo de un recurso como el agua. Estas son algunas condiciones estructurales que han sido la base para la conflictividad que se ha vivido en el distrito, máxime si a esto se suma una ambivalencia institucional en cuanto al manejo de estos proyectos.

De este modo, la conflictividad por las urbanizaciones cerradas en San Miguel apareció acompañado por la búsqueda de instaurar dos plantas de asfalto (CONANSA y Quebradores Río Frío S.A) cerca de tales proyectos, donde la acción colectiva vecinal ha logrado postergarlos, pero hasta la fecha no han sido archivados.(Salas, 2012; Novo, 2013). ${ }^{11}$ Se debe sumar el caso de la empresa Trigal, que se dedica a almacenar contenedores y que invadió el cauce del Río Virilla incluso desviándolo ${ }^{12}$, caso que data desde el 2008 y que a la fecha no ha sido resuelto, mientras la empresa sigue con sus operaciones (Fracción legislativa del PAC, 2017).

Para el caso de los proyectos urbanísticos se conoce de ocho proyectos ${ }^{13}$ de los cuales algunos se están construyendo mientras que otros se encuentran en procesos administrativos. Esto ha generado una serie de acciones colectivas que se agudizaron en el 2016, pero que tienen sus antecedentes en diciembre del 2012 al conformar un comité que se encargara de investigar lo relacionado con tal proceso: El CCCRV.

Nos dimos a la tarea de, cómo podíamos, empezar a regar la bola, empezar a contactar la gente, vecinos, vecinas y hacer una reunión. (...) Eso nos obligó a salir de nuestras casas y conocer a nuestros vecinos y vecinas. (...) En una reunión en el colegio Lincoln los vecinos nos decían que sería importante que se conformara un comité que sea el cual tome las decisiones, que sea el que decida que esfuerzos llevar a cabo, que líneas trabajar y después nos informan a la comunidad. (...) La comunidad designó a las personas y así fue como conformamos el comité, muchos ni nos conocíamos. (Lideresa comunal del CCCRV, comunicación personal, 1 de junio, 2018)

El CCCRV descubrió posteriormente los diversos proyectos urbanísticos, donde la principal carta de oposición es que la Municipalidad de Santo Domingo no cuenta con el Plan Regulador, por lo que queda sujeta a la legislación general relacionada con el tema. Entre esto se encuentra el decreto ejecutivo $\mathrm{N}^{\circ}$ 25902-MIVAH-MP-MINAE que establece un área de control urbanístico en el que se crea una Zona Especial 
de Protección. De este modo, se permiten urbanizaciones cerradas solamente en la cabecera de distrito y 200 metros a la redonda, por lo que fuera de este perímetro es recomendable solamente una casa por finca para mantener el carácter agrícola y ganadero del distrito.

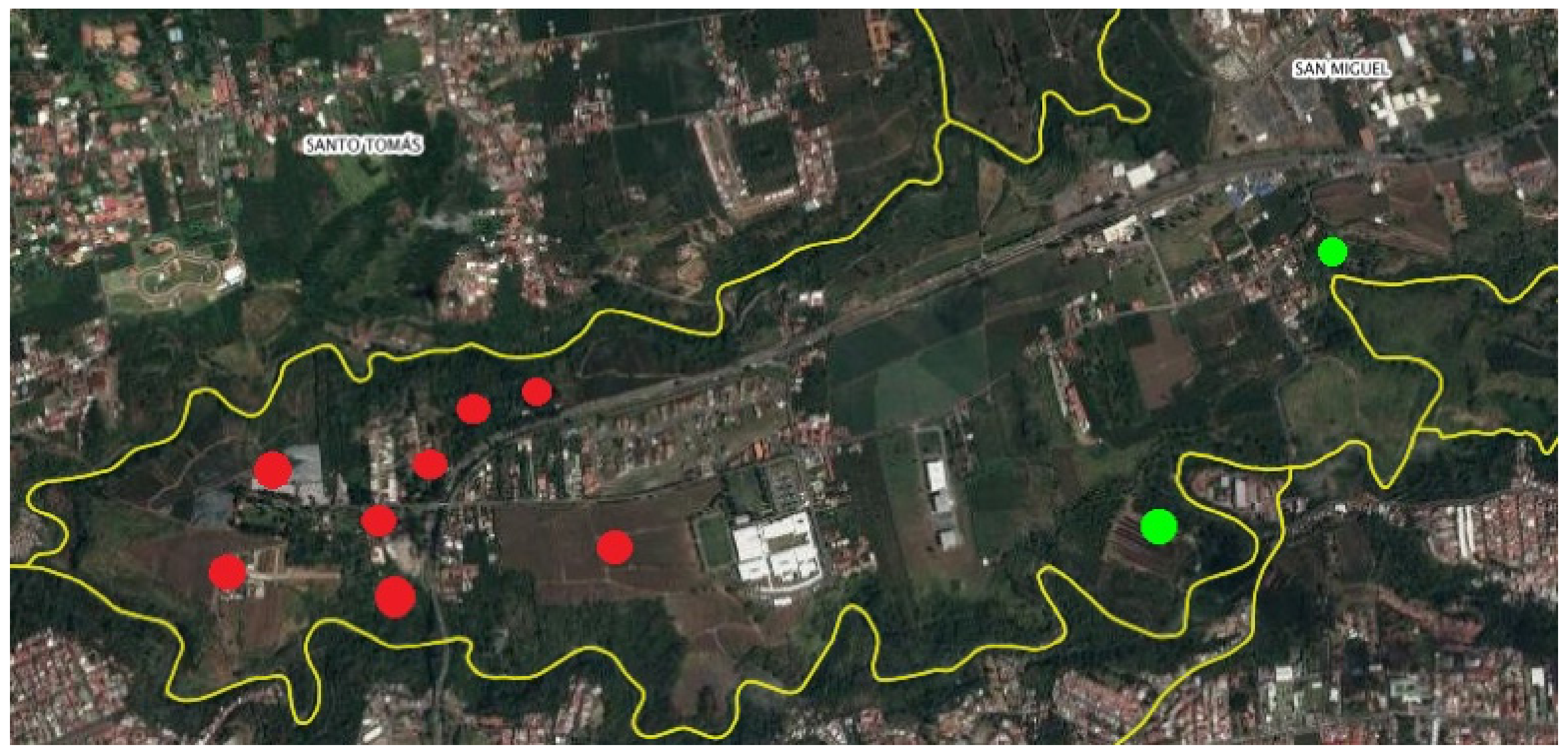

Figura 2. Imagen satelital con los proyectos de urbanización y de plantas de asfalto en disputa en San Miguel (2018). En rojo: Proyectos Urbanísticos. En verde: Plantas de asfalto. Fuente: elaboración propia con base en los mapas sociales del INEC e información suministrada por el CCCRV.
14 Cronología elaborada a partir de la entrevista con la lideresa comunal, el blog del Comité Bandera Azul Ecológica San Miguel, actas municipales y notas periodísticas.
Los proyectos denunciados por los vecinos se encuentran ubicados en la Zona Especial de Protección, los cuales se aprecian en la Figura 2. A esto se debe sumar la zona de recarga acuífera ya mencionada, así como la demanda de agua de la que requieren estos proyectos lo que entra en contradicción con el problema de abastecimiento que ha existido en el distrito. Asimismo, el Servicio Nacional de Aguas Subterráneas, Riego y Avenamiento (SENARA) desde hace una década advirtió de una sobreexplotación de los acuíferos Barva y Colima, por lo que proyectos urbanísticos se tornarían peligrosos para la recarga de los acuíferos (Angulo \& Oviedo, 2008).

De este modo, al repasar cronológicamente la conflictividad en torno a este caso se presentan los siguientes acontecimientos principales ${ }^{14}$ :

En noviembre del 2012, los vecinos son alertados por la posibilidad de la construcción de una planta de asfalto. Al revisar expedientes en SETENA se dan cuenta que son dos. Esto hace que para diciembre de tal año se configure el CCCRV.

A partir de ese momento, se organizaron manifestaciones a las afueras de la Municipalidad de Santo Domingo, así como la repartición de volantes y reuniones comunales que dieron como resultado un acuerdo por parte del Concejo Municipal en enero del 2013, en el que se restringe la aprobación de permisos de actividades de carácter industrial u otros proyectos que atenten contra el recurso hídrico de la zona.

Sin embargo, para setiembre del 2014 un abogado de la alcaldía aduce que el acuerdo atenta contra las restricciones legítimas de la propiedad privada según el artículo 45 de la Constitución Política y el artículo 17 de la Ley de Planificación Urbana, por lo que el Concejo Municipal decide desestimar el acuerdo logrado con los vecinos. La posibilidad de proyectos industriales y urbanísticos cobra auge nuevamente.

El año 2015 dio matices de una mejora en la gestión del agua, esto porque en marzo de este año entró en funcionamiento una planta potabilizadora por parte de la municipalidad para abastecer los distritos de San Miguel, Pará, Paracito y Tures.

Aun así, la conflictividad por la gestión del recurso se reactivó en abril de 2016 a raíz de la aprobación de dos proyectos urbanísticos, lo cual generó una serie de manifestaciones a las afueras de la municipalidad, volanteo y reuniones comunales.

La acción colectiva causó que el Concejo Municipal emitiera una moratoria de dos meses para otorgar permisos de este tipo a partir de mayo del 2016, lo cual coin- 
Figura 3. Imagen de una protesta a las afueras de la Municipalidad de Santo Domingo el día 25 de abril de 2016. Fuente: (Araya, 2016, s.f) cidió con el cambio de administración a la que se le sugirió realizar valoraciones técnicas y legales en cuanto a esta temática.

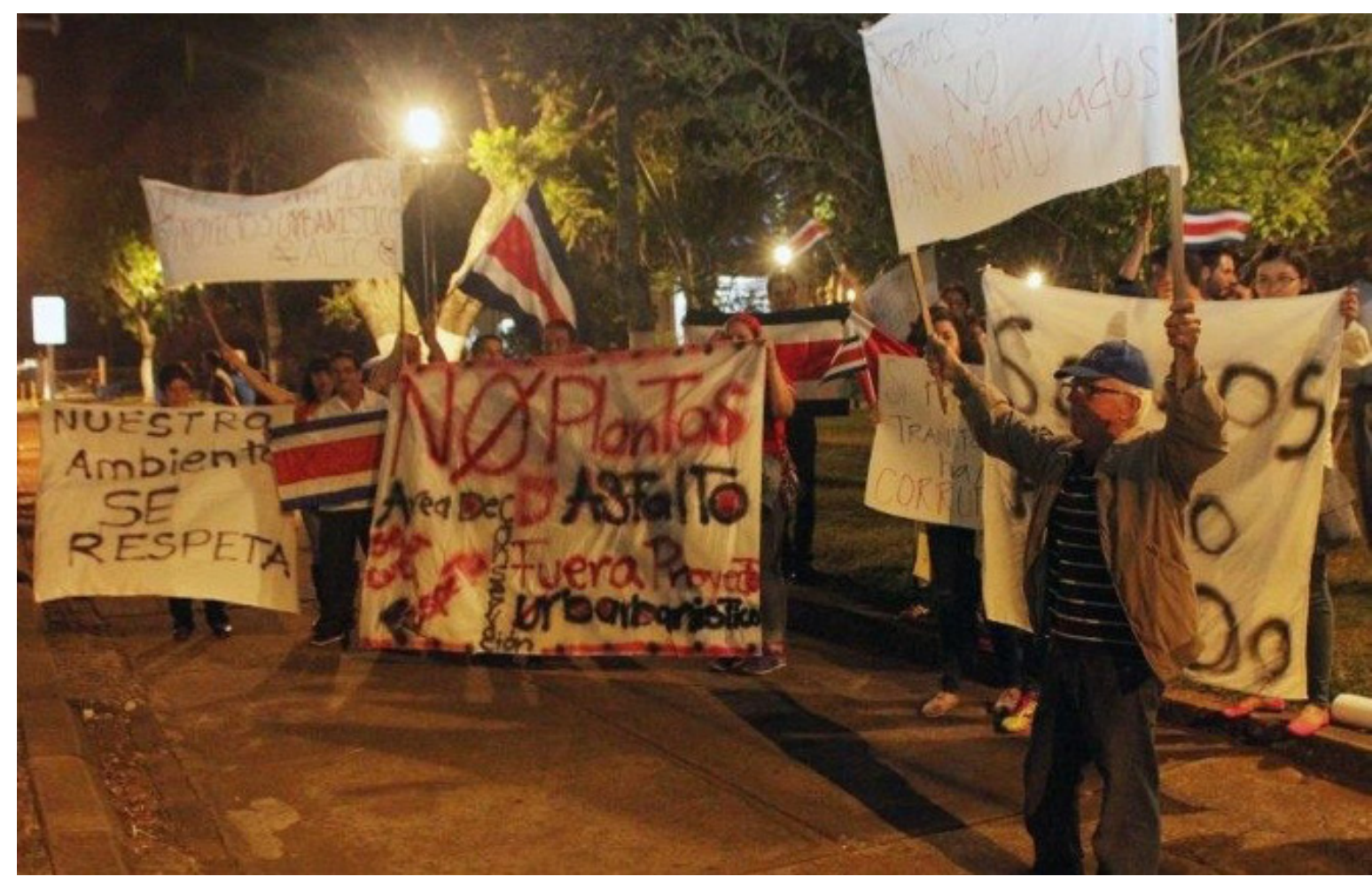

Todo este proceso dejó como resultado cuatro proyectos aprobados de los ocho denunciados: Vista Catedral, Hacienda Lincoln, Los Eucaliptos y Tecknau. Vista Catedral ya se encuentra en funcionamiento, el cual es un condominio que consta de 75 casas de $150 \mathrm{~m}^{2} .{ }^{15}$ De igual manera, el condominio Tecknau consta de 52 casas, cada una posee tres habitaciones, terrazas, áreas comunes y se promociona como un sitio cercano a San José, Tibás y Moravia. ${ }^{16}$

Los dos restantes se encuentran en fase de construcción; el proyecto los Eucaliptos consiste en seis torres de apartamentos de ocho pisos ${ }^{17}$, mientras que Hacienda Lincoln es un condominio que constará con construcciones verticales y horizontales con 124 casas. ${ }^{18}$ Es así como la suma de todos estos proyectos se torna considerable en un distrito que alberga menos de 7000 habitantes.

Por su parte, con respecto a las urbanizaciones los Eucaliptos y Hacienda Lincoln, se encuentra la particularidad de un acuerdo con la municipalidad para la concesión de un pozo. Tal pozo era administrado por el acueducto municipal donde se obtenían 4 litros de agua por segundo. Sin embargo, una vez que se aprobaron tales proyectos urbanísticos se decidió acordar con los desarrolladores ampliar el pozo para poder obtener más agua (22 l/s) donde Los Eucaliptos se encargó de este proceso técnico. Por su parte, Hacienda Lincoln se encargó de mejorar las tuberías y el sistema eléctrico del mismo. La condición del acuerdo fue que el pozo dotará de agua a los proyectos cuando estén finalizados y se suministrará de agua al acueducto municipal solo en situaciones de emergencia. ${ }^{19}$

Tales proyectos, acompañados con la permisividad de la municipalidad, así como los decretos que contradicen las acciones municipales hacen que los vecinos cuestionen lo que ocurre en el distrito: "Para mí en lo personal es esa incongruencia entre lo que se define para esta zona con respecto a estos proyectos ¿si esta es una Zona de Protección Especial definido por ley porque diablos le meten todo esto encima? ¿Qué estamos protegiendo?" (Líder Comunal del CCCRV, comunicación personal, 18 de julio, 2018)

Además, este vecino aduce que la Municipalidad de Santo Domingo es la principal institución cuestionable en este proceso,

(...) porque ahí empieza todo, si yo te doy el uso de suelo es porque ya te lo permití. Si uno va a sacar un uso de suelo y le preguntan qué va a hacer si va a construir una casa o va a ampliar, le dicen que es una zona de protección especial, pero cuando uno ve esos proyectos de 700 apartamentos uno dice ¿qué pasa? (Líder Comunal del CCCRV, comunicación personal, 18 de julio, 2018)

Con él coincide la lideresa entrevistada, esto con respecto al papel de la municipalidad, además de que SETENA es otra institución involucrada al otorgar la viabilidad ambiental de los proyectos. Así: 
(...) hemos topado gente que aporta positivamente como gente que ha entorpecido el proceso. Gente que parece aprovechar la vía legal para favorecer a la propiedad privada en detrimento del bien público, desde alcaldes, alcaldesas, profesionales en derecho $\mathrm{e}$ ingenieros etc., y así en las instituciones, dependiendo de quién esté, hay más o menos apertura para tocar los temas. (Lideresa comunal del CCCRV, comunicación personal, 1 de junio, 2018)

Por otra parte, un vecino de la localidad que es ex regidor del municipio, se posiciona a favor de estas urbanizaciones ya que:

(...) todo el mundo tiene derecho a crear su desarrollo habitacional, siempre con los requisitos que estipula el plan regulador, que ese plan ha costado mucho porque ha habido mucha traba de parte de gente que de manera agazapada ha impedido que sea una realidad. Los proyectos de urbanización valen la pena, pero a veces uno se queda receloso sobre eso. (Ex regidor-vecino de San Miguel, 28 de octubre, 2018)

Esto refleja que la oposición vecinal a estas urbanizaciones no es homogénea, no obstante, este vecino que está a favor de igual manera recalca la importancia de una regulación en torno a estos esquemas habitacionales. Aun así, aliviana la carga de responsabilidad del municipio al aducir que son otros intereses los que han frenado la culminación del Plan Regulador.

\section{El agua entre lo público y lo privado: reflexiones finales.}

El caso expuesto refleja diferentes aristas de la gestión del agua, ya que no es solo una cuestión de abastecimiento y potabilidad, sino que, para que se cumplan estos dos principios, en primer lugar, debe existir protección sobre las fuentes del recurso, así como en la forma de obtener el líquido.

De este modo la conflictividad en San Miguel se ha desplazado entre estas variables, donde las mejoras en cuanto a la potabilidad del agua no han sido sinónimo de una resolución del conflicto, ya que la preocupación por los acuíferos, los pozos, los ríos y el abastecimiento a futuro, también han formado parte de la agenda comunal.

Aun así, tales preocupaciones y las acciones realizadas no bastaron para detener las construcciones valoradas negativamente. Incluso la manera en que se ha gestionado el recurso se ha convertido en un caso que cuestiona las barreras entre la gestión tradicional del agua ligada a lo público y la gestión privada de la misma. Esto obliga a repensar las conceptualizaciones de gestión del agua (público, privado, comunal), ya que en un caso como éste se han presentado de una forma entremezclada.

En esta vía, las dificultades que ha tenido la Municipalidad de Santo Domingo para gestionar el agua, el entramado institucional-legal relacionado con el tema, así como la asimetría de poder presente entre los actores involucrados decantaron en acuerdos de carácter híbrido entre lo público y lo privado como el caso del pozo que ahora es administrado por dos desarrolladores de proyectos urbanísticos. Esto refleja la argumentación de Rabotnikof (2005) mencionada anteriormente, sobre como agrupaciones de carácter privado han asumido el manejo de lo público ante el deterioro del Estado y sus instituciones.

Ante tal panorama, también se encuentran los intentos por parte de actores comunales para posicionar elementos de una gestión social del agua en la que se busca insertar elementos de cooperación y solidaridad al intentar proteger los cuerpos de agua, aun cuando estos actores se encuentran en desigualdad de recursos (económicos, legales, incidencia en decisiones políticas) en relación con distintos actores económicos que han incidido en la gestión pública. Un ejemplo claro ha sido la forma en que los actores comunales han logrado moratorias y restricciones que han sido boicoteadas por presión de privados ante el Concejo Municipal (Acta municipal; sesión 8 de setiembre del 2014).

Tales tensiones se tradujeron en una conflictividad socioambiental con diferentes formas de interpretar, percibir y valorar el agua, lo cual se evidenció en una fricción entre distintos actores, sobre todo con una serie de acciones colectivas en la esfera pública dirigidas principalmente hacia la Municipalidad de Santo Domingo. En esta línea, el caso permite caracterizar tres posicionamientos básicos a partir de las valoraciones que se tiene del agua: 
Figura 4. Posicionamientos generales en torno a la gestión del agua en San Miguel. Fuente: Elaboración propia.
$20 \quad$ Un ejemplo es la conflictividad en torno a la Loma Salitral en Desamparados, la cual es una zona protegida de la GAM. Sin embargo, la Municipalidad de Desamparados ha otorgado permisos para que se construya una urbanización cerrada de 500 casas a pesar de que el Plan Regulador prohíbe construcciones de este tipo en la zona. Esto ha generado resistencia por parte de grupos comunales y ambientalistas (Noguera, 2018).

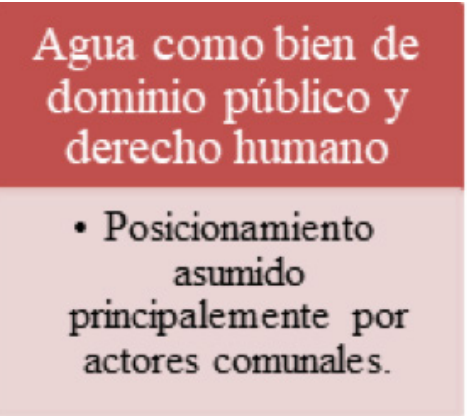

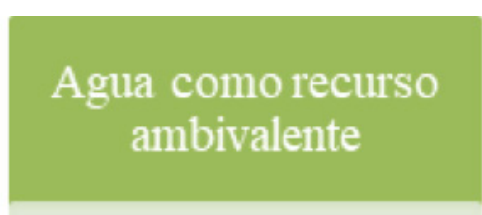

- Posicionamiento asumido

principalmente por la Municipalidad
Agua como recurso

para fines

económicos

- Posicionamiento asumido por los desarrolladores de los proyectos urbanisticos

La Figura 4 refleja la valoración que distintos actores comunales hacen del agua, la cual se liga a lo público donde el acceso y la disponibilidad del líquido para los habitantes de la zona son primordiales: "Para mí es un bien de dominio público porque es un elemento que nos da la naturaleza y es de acceso para todos y todas, nadie debería apropiarse de esta. Es algo que está ahí, que el mundo nos da y es para vivirlo y disfrutarlo" (Lideresa comunal del CCCRV, comunicación personal, 1 de junio, 2018).

En contra parte, el agua está al servicio de las actividades de acumulación de excedente, ya que para un proyecto inmobiliario en el que se busca generar ganancia a partir de la venta 0 alquiler de casas que sobrepasen la inversión inicial, la disponibilidad de un recurso como el agua es fundamental. Esto no quiere decir que la posición de los desarrolladores con respecto al recurso sea esa únicamente, no obstante, se ha visto reflejada en los acuerdos logrados para obtenerlo.

Asimismo, las concesiones a estas construcciones para administrar el agua refleja una situación de acaparamiento de oportunidades, al presentarse situaciones monopólicas con respecto al acceso a recursos valorados positivamente (Pérez-Saínz, 2014), en este caso, el agua. El caso más claro es el del pozo administrado directamente por Los Eucaliptos y Hacienda Lincoln, donde los pobladores ajenos a estas urbanizaciones cerradas quedan excluidos parcialmente del acceso a dicha fuente.

Por su parte, el papel asumido por la Municipalidad de Santo Domingo es de carácter ambivalente, ya que, a pesar de lograr mejoras en el acueducto al otorgar permisos de construcción en la zona en disputa, los cuerpos de agua son desprotegidos. Lo mismo sucede con la legislación y otras instituciones (SETENA, SENARA, AyA, Ministerio de Salud) que intentan proteger el recurso, pero que la descoordinación deja vacíos de los que se valen actores con otros intereses.

Por lo tanto, esta tipología de posicionamientos y los factores asociados a estos reflejan la proposición de la ecología política en la que las tensiones en torno a la naturaleza están mediadas por cuestiones económicas (interés inmobiliario) y políticas (complacencia del gobierno local) que se reflejan en disputas en ámbitos locales.

Además, el caso expuesto forma parte de dinámicas regionales a nivel provincial ya que existen zonas como San Rafael, San Isidro, Santa Bárbara y Barva (Angulo \& Oviedo, 2008; Montero, 2016) que han presentado problemáticas por desabastecimiento de agua, donde proyectos urbanísticos ambiciosos agudizan tales preocupaciones. Es asi como este artículo insta a continuar una línea investigativa que analice estas situaciones a nivel regional, vislumbrando semejanzas y particularidades.

Finalmente, los acontecimientos de San Miguel reflejan una serie de retos en torno al ordenamiento territorial. La ausencia de un Plan Regulador para Santo Domingo es parte importante de las tensiones analizadas, al no haber claridad sobre la distribución del uso de suelo del cantón. Esto conduce a basarse en diferentes criterios técnicos y mecanismos legales como decretos, leyes, reglamentos, etc., que tienden a no ser homogéneos, generando vacíos que son aprovechados por diferentes actores.

Sin embargo, la existencia de tal plan no acabaría inmediatamente con este tipo de problemáticas, esto porque de fondo existen actividades que generan renta, con intereses de actores con capital económico y social que pueden esquivar este tipo de mecanismos ${ }^{20}$ Asimismo, las motivaciones y percepciones en torno al territorio y la naturaleza son variadas, lo que puede desencadenar en conflictividades a pesar de una regulación territorial. 


\section{Bibliografía}

Agüero, J.; Pujol, R. \& Pérez, E. (2014). Actividad económica, características sociales y demanda de transporte: Una exploración de los patrones de viajes residenciatrabajo en el censo de 2011. En Instituto Nacional de Estadística y Censos (ed). Costa Rica a la Luz del Censo del 2011. San José, Costa Rica.

Alpízar, F. (2012). ¿Democracia ecológica? Las instituciones, la participación política y las contiendas por el agua en Costa Rica (1821-2010) (Tesis de doctorado). Universidad Complutense de Madrid.

Alvarado, X.; Avendaño, S.; Blanco, C.\& Rodríguez, G. (2004). Análisis de política pública con relación al Anteproyecto de Ley del Recurso Hídrico en Costa Rica: el acceso al agua como derecho ciudadano(Seminario de Graduación de Licenciatura). Universidad de Costa Rica.

Angulo, F. (2015). Informe final. Gestión del recurso hídrico y saneamiento en Costa Rica. Vigésimo primer Informe Estado de la Nación en Desarrollo Humano Sostenible. San José, Costa Rica.

Angulo, F.\& Oviedo, E. (2008, 8 de marzo). Falta de agua amenaza auge urbano en el Valle Central. La Nación. Recuperado de: http://wvw.nacion.com/ln_ee/2008/ marzo/08/pais1453261.html

Araya, J. (2016, 4 de mayo). "Defensores de acuíferos logran moratoria de desarrollos inmobiliarios." Semanario Universidad. Recuperado de: https:// semanariouniversidad.com/pais/defensores-acuiferos-logran-moratoriadesarrollos-inmobiliarios/

Astorga, A. (2011). Ordenamiento territorial en Costa Rica.(Reporte No. 17). San José, Costa Rica: Estado de la Nación.

Bolaños,C. (2016). Conflictos socioambientales por la gestión del agua: el caso de la comunidad de Playa Potrero, Guanacaste (Trabajo final de licenciatura). Universidad de Costa Rica.

Castells, M. (2009). Comunicación y poder. Madrid: Alianza Editorial.

Clare, P; Meléndez, M. (2012). Articulaciones entre ecología política, geografía histórica e historia ambiental: paisaje y poder. Revista Espaciotiempo. Recuperado de: http://repositorios.cihac.fcs.ucr.ac.cr/repositorio/bitstream/123456789/473/1/ ClareP\%20en\%20EspacioTiempo.pdf

Fenell, M. (2013). Las concesiones de la prestación del servicio de agua potable por parte de sujetos privados desde la perspectiva del derecho ambiental: El caso Sardinal de Carrillo, Guanacaste (Tesis de Licenciatura). Facultad de Derecho, Universidad de Costa Rica.

Fonseca, A. (2011, 4 de octubre). Pobladores de Santo Domingo achacan al agua brote de diarrea. La Nación. Recuperado de:https://www.nacion.com/el-pais/servicios/ pobladores-de-santo-domingo-achacan-al-agua-brote-de-diarrea/5JWKHUUKQB AZHIFUFKF3IOXTOQ/story/

Fonseca, A. (2011, 5 de octubre). 15.000 personas toman agua con materia fecal en

Santo Domingo. La Nación. Recuperado de: https://www.nacion.com/el-pais/ servicios/15-000-personas-toman-agua-con-materia-fecal-en-santo-domingo/ Q57SKVD3RRFFTKWBXOZR7BNHTA/story/

Foucault, M. El sujeto y el poder. (1988). Revista Mexicana de Sociología, 50(3),3-20. Recuperado de: http://links.jstor.org/sici?sici=0188-2503\%28198807\%2F09\%295 0\%3A3\%3C3\%3AESYEP\%3E2.0.CO\%3B2-A

Fracción Legislativa PAC. (2017). PAC aboga por investigar empresa que causó grave daño ambiental en el río Virilla. Recuperado de: https://pac.cr/pac-aboga-porinvestigar-empresa-que-causo-grave-dano-ambiental-en-el-rio-virilla/

Gudynas, E. (2014). Conflictos y extractivismos: conceptos, contenidos y dinámicas. DECURSOS, 79-115. Universidad Mayor San Simón, Cochabamba. 
Recuperado de: http://extractivismo.com/wp-content/uploads/2016/07/ GudynasConflictosExtractivismosConceptosDecs14.pdf

INEC. (2011). Censo Poblacional. Recuperado de: http://www.inec.go.cr/Web/Home/ GeneradorPagina.aspx

Libro de Actas Municipales de 2014. Municipalidad de Santo Domingo, Heredia, Costa Rica. Actas número: 328, 360.

Madrigal-Solís, H., Fonseca-Sánchez, A., Núñez-Solís, C., \& Gómez-Cruz, A. (2017). Amenaza del agua subterránea en el sector norte del acuífero Barva, Heredia, Costa Rica. Tecnología y Ciencias del Agua, 5(6), 103-118. Universidad Nacional.

Monge, C. (1987). Efectos del proceso de urbanización sobre el recurso agua en la unidad geográfica Barva, Heredia, Costa Rica (Tesis de licenciatura). Universidad de Costa Rica.

Montagnini, F. (2018). Gestión del agua en Costa Rica: Estudio de caso de la conflictividad en San Miguel de Santo Domingo de Heredia (2011-2018) (Tesis de licenciatura). Universidad de Costa Rica.

Montero, D. (2016). Problemas en la capital del agua: el caso del conflicto por el desabastecimiento de agua potable durante el 2014 en el cantón de Barva de Heredia (Tesis de licenciatura). Universidad de Costa Rica.

MOPT (2010). Manual de especificaciones generales para la construcción de carreteras, caminos y puentes. CR-2010.Recuperado de: https://www.mopt. go.cr/wps/wcm/connect/28a27ca9-2ec2-49ae-838c-6f89e21d43b4/CR-2010. pdf?MOD=AJPERES

Navas, G.\&Cuvi, N. (2015). Análisis de un conflicto socioambiental por agua y turismo en Sardinal, Costa Rica. Revista de Ciencias Sociales, 150, 109-124. Universidad de Costa Rica.

Novo, Y. (2013, 28 de enero). Vecinos de Santo Domingo de Heredia insisten en el principio precautorio ante construcción de planta asfáltica. Crhoy.com. Recuperado de: http://www.crhoy.com/archivo/vecinos-de-santo-domingo-de-heredia-insistenen-el-principio-precautorio-ante-construccion-de-planta-asfaltica/nacionales/

Oviedo, S. (2015, 6 de marzo). Municipalidad de Santo Domingo invirtió $ф 765$ millones en planta potabilizadora de agua. La Nación.Recuperado de: https://www.nacion. com/el-pais/servicios/municipalidad-de-santo-domingo-invirtio-c-765-millonesen-planta-potabilizadora-de-agua/VPWMZPLHNZHP5H7D3REYTAPKWE/story/

Pérez-Sáinz, J. (2014). Mercados y bárbaros: Persistencia de las desigualdades de excedente en América Latina. San José: FLACSO.

Pérez, J.P. \& Alvarado, K. (2012). Globalización y nuevos ejes de acumulación en Costa Rica. (Texto inédito) Recuperado de: https://studylib.es/doc/7923921/ globalizaci\%C3\%B3n-y-ejes-de-acumulaci\%C3\%B3n-en-costa-rica

Rabotnikof, N. (2005). En busca de un lugar común: el espacio público en la teoría política contemporánea. Instituto de Investigaciones Filosóficas: Universidad Nacional Autónoma de México.

Ragin, C. (2007). La construcción de la investigación social. Introducción a sus métodos y diversidad. Colombia: Siglo del hombre Editores.

Ramírez, A. (2008). Conflictos socioambientales y recursos hídricos en Guanacaste; una descripción desde el cambio en el estilo de desarrollo (1997-2006). Anuario de Estudios Centroamericanos 33-34, 359-385. Universidad de Costa Rica.

Salas, L. (2012, 15 de diciembre). Vecinos de Santo Domingo se oponen a construcción de planta asfáltica en la zona. Crhoy.com. Recuperado de: http://www.crhoy. com/archivo/vecinos-de-santo-domingo-se-oponen-a-construccion-de-plantaasfaltica-en-la-zonal

Silva, J. (2016). El movimiento social por el Agua en Sardinal de Guanacaste (Tesis de licenciatura). Universidad de Costa Rica.

Solís, D. (2017, 23 de mayo). Santo Domingo y su zona especial de protección. Periódico 
El Guacho. Recuperado de: http://periodicoelguacho.com/santo-domingo-deheredia-y-su-zona-especial-de-proteccion/

Vargas, C. (2006). Evaluación de la disponibilidad de los recursos hídricos (superficiales y subterráneos) para el cantón de Santo Domingo de Heredia (Tesis Licenciatura). Universidad de Costa Rica, Costa Rica.

Walter, M. (2009). Conflictos ambientales, socioambientales, ecológico distributivos, de contenido ambiental... Reflexionando sobre enfoques y definiciones. CIP-Ecosocial, Madrid. Obtenido de: http://crana.org/themed/crana/files/ docs/252/180/2009_conflictos_ambientales_enfoques_definiciones.pdf

Weber, M. (2002). Economía y sociedad. Esbozo de sociología comprensiva. Madrid: Fondo de Cultura Económica. 